\title{
Perspective authors' response: Patterns of neural stem and progenitor cell division may underlie evolutionary cortical expansion
}

\author{
Arnold R. Kriegstein*, Verónica Martínez Cerdeño ${ }^{\ddagger}$ and Stephen C. Noctor $§$
}

A significant body of work has emerged from multiple laboratories over the past several years demonstrating cortical neurogenesis in the embryonic subventricular zone (SVZ). The role of SVZ precursor cells as a major source of cortical neurogenesis was not appreciated at the time that Van Essen formulated his hypotheses on cortical expansion and gyrification ${ }^{1}$. Our basic premise concerns the potential significance of a newly described neuronal progenitor cell type found in the SVZ, referred to as an intermediate progenitor (IP) cell. IP cells have been best characterized in rodents, but similar cells have been described in primate cortex ${ }^{2}$. In fact, the primate ventricular zone becomes very thin at the onset of cortical neurogenesis, and the SVZ might be the major source of neurogenesis in the primate visual system ${ }^{2}$. Our paper speculates on the impact of IP cell neurogenesis on features of cortical development, particularly the generation of upper cortical layers, and cortical expansion. Our remarks about the possible evolutionary roles of IP cells owe much to the observation that the SVZ is practically non-existent in the simple three-layered reptilian cortex ${ }^{3}$, is appreciable in rodents and is extremely large in primates. If the primate embryonic SVZ harbours large numbers of IP cells, as we propose, the production of neurons from this region cannot be overlooked in models of cortical development and cortical expansion. It is not our intention to dismiss previous hypotheses, but to suggest that a revision that incorporates recent findings might be appropriate.

Dr Van Essen faults our work as reversing cause and effect, claiming that the width of the SVZ is dependent on the presence of axonal fibres, and thereby suggesting that it is not correlated with cortical neurogenesis. But one could argue that this is putting the cart before the horse, as the embryonic SVZ is a neurogenic compartment that produces the very cortical neurons that later send axonal projections through various cortical structures. Cause-and-effect relationships between cell generation and cell maturation will need to be examined through experimentation and manipulation to determine the precise relationship between the size of the SVZ and cortical expansion. For example, absence of sonic hedgehog $(\mathrm{SHH})$ during cerebellar development results in a smooth, unfoliated cerebellar cortex in which sulci are completely lacking. By contrast, overexpression of SHH produces extra foliation and tangentially expanded cerebellar lobules ${ }^{4}$. Interestingly, $\mathrm{SHH}$ appears to mediate this effect by stimulating the proliferation of precursor cells in the external granular layer ${ }^{4}$, providing a strong link between the degree of cortical folding and the levels of proliferation. These findings support our concept that proliferation might be relevant to gyral formation in the cerebral cortex as well.

Dr Van Essen is correct to point out that in our figure $3 \mathrm{~b}$, the brackets include stratified transitional fields, and should not have been identified as the SVZ per se. However, during stages of cortical neurogenesis in the rodent, the stratified transitional fields overlap with the SVZ and with the intermediate zone $^{5}$, regions where many IP cells reside. Van Essen implies that neurogenesis does not occur in the intermediate zone. However, as pointed out by Altman and Bayer in the work cited by Van Essen, we know comparatively little about cortical neurogenesis in humans, and in the absence of hard data the best we can do is "extrapolate from the combined experimentally established sequence of cortical neurogenesis and transitional field stratification in rats to humans" (REF. 6). Most important for the arguments we make in our hypothesis, IP cells in rodents are not restricted to the SVZ, but are also found in the outer margin of the ventricular zone and in the intermediate zone (REF. 7 and S.C.N., V.M.-C. and A.R.K., unpublished observations). The development of markers for human IP cells will aid in determining to what extent IP cells are present in the human stratified fields, and to what extent cortical neurogenesis occurs in these compartments.
An understanding of the mechanism that produces the human smooth cortex disorder lissencephaly might be expected to shed light on our discussion. In classical lissencephaly the cortex is reduced to an agyric four-layered structure. Mutations in the LIS1 gene are one of the causes of classical lissencephaly ${ }^{8}$, and the effects of reducing LIS1 protein levels have been studied in the developing mouse cortex. In addition to defects in neuronal migration, IP cell production is severely reduced ${ }^{9}$, but there are also significant reductions in radial glial cell division and abnormalities in axon formation ${ }^{9,10}$. Not only do these results not rule out the models of cortical expansion under discussion, they could even be interpreted as supporting each. In conclusion, we believe that our hypothesis does not contradict that of Van Essen. Instead, we believe that existing hypotheses must account for emerging data, and be flexible enough to incorporate new findings concerning cortical histogenesis.

\section{Arnold R. Kriegstein is at the Institute for Regeneration Medicine, Department of Neurology, University of California, San Francisco, 513 Parnassus Ave, San Francisco, California 94143, USA.}

Verónica Martinez Cerdeño is at the Department of Neurology, University of California, San Francisco, 513 Parnassus Ave, San Francisco, California 94143.

USA.

Stephen C. Noctor is at The MIND Institute, Department of Psychiatry and Behavioral Sciences, University of California, Davis, 2825 50th Street, Sacramento, California 95817, USA.

1. Van Essen, D. C. A tension-based theory of morphogenesis and compact wiring in the central nervous system. Nature 385, 313-318 (1997).

2. Smart, I. H., Dehay, C., Giroud, P., Berland, M. \& Kennedy, H. Unique morphological features of the proliferative zones and postmitotic compartments of the neural epithelium giving rise to striate and extrastriate cortex in the monkey. Cereb. Cortex 12 37-53 (2002)

3. Martínez-Cerdeño, V., Noctor, S. C. \& Kriegstein, A. R. The role of intermediate progenitor cells in the evolutionary expansion of the cerebral cortex. Cereb. Cortex 16 (Suppl. 1), i152-i161 (2006).

4. Corrales, J. D., Blaess, S., Mahoney, E. M. \& Joyner, A. L. The level of sonic hedgehog signaling regulates the complexity of cerebellar foliation. Development 133, 1811-1821 (2006)

5. Bayer, S. A. \& Altman, J. Neocortical Development. 18 (Raven, New York, 1991).

6. Altman, J. \& Bayer, S. A. Regional differences in the stratified transitional field and the honeycomb matrix of the developing human cerebral cortex.

J. Neurocytol. 31, 613-632 (2002).

7. Takahashi, T., Nowakowski, R. S. \& Caviness, V. S. Jr. Early ontogeny of the secondary proliferative population of the embryonic murine cerebral wall. J. Neurosci. 15, 6058-6068 (1995).

8. Pilz, D. T. et al. LIS1 and XLIS (DCX) mutations cause most classical lissencephaly, but different patterns of malformation. Hum. Mol. Genet. 7, 2029-2037 (1998)

9. Tsai, J. W., Chen, Y., Kriegstein, A. R. \& Vallee, R. B. LIS1 RNA interference blocks neural stem cell division, morphogenesis, and motility at multiple stages. J. Cell Biol. 170, 935-945 (2005)

10. Gambello, M. J. et al. Multiple dose-dependent effects of Lis 1 on cerebral cortical development. J. Neurosci. 23, 1719-1729 (2003). 